\title{
INFECTIVE ENDOCARDITIS CAUSED BY GROUP G STREPTOCOCCUS WITH MULTIPLE CEREBRAL EMBOLI
}

\author{
Ilknur Erdem ${ }^{1}$, Pasa Goktas ${ }^{1}$, Refik Demirtunc ${ }^{2}$, Aysun Erdem ${ }^{3}$ \\ Haydarpasa Numune Training and Research Hospital, Uskudar, Istanbul: Clinic of Infectious Disease and Clinical \\ Microbiology ${ }^{1}$, Clinic of Internal Medicine ${ }^{2}$; Maltepe University, Medical Faculty, Istanbul: Department of Cardiology ${ }^{3}$
}

\begin{abstract}
Summary: The group G streptococcal endocarditis is a rare form of infective endocarditis. In this form of infective endocarditis, serious neurological complications most commonly develop. We reported this case because of its being an unusual form of infective endocarditis that was caused by Group G Streptococcus. We also reviewed the literature. The patient was admitted to infectious disease service with a presumptive diagnosis of central nervous system infection. Blood cultures were positive for group $\mathrm{G}$ streptococcus. There was a mass on the posterior surface of the mitral valve which was $2 \mathrm{x} 2.5 \mathrm{~cm}$ in length on the echocardiography. In the cranial computerized tomography of our patient, slightly increased contrast media uptake was observed in the both parietal lobes, in the both frontal lobes, and in the anterior areas of right occipital lobe. Therefore, this case was assumed as infective endocarditis caused by group $\mathrm{G}$ streptococcus with multiple cerebral emboli. Ceftriaxone was given for 4 weeks and gentamicin was given for 2 weeks, and progressive improvement of the patient's condition was seen.
\end{abstract}

Key words: Infective endocarditis; Cerebral emboli; Group G streptococcus

\section{Introduction}

Infective endocarditis is an infection of the endocardial structures, including the cardiac valves and mural endocardium. There are both persistent bacteremia and anatomic lesions of the valves in infective endocarditis. Infective endocarditis may result in life-threating complications. Therefore, precise clinical and microbiologic diagnosis is important and appropriate antibiotic therapy must be considered. In recent years, advances both in clinical microbiology and in the imaging techniques, especially transthoracic and transesophageal echocardiography, have made the diagnosis of infective endocarditis more accurate. Due to these recent developments Durack et al. proposed a new diagnostic criteria that known as Duke criteria in 1994. Duke criterias were revised in year 2000 (2,5,10,11,13).

Many different microorganisms may cause infective endocarditis, but the majority of cases are caused by streptococci, particulary Streptococcus viridans. Infective endocarditis caused by beta hemolytic streptococci is infrequently seen. The frequency of group $G$ beta hemolytic streptococcal endocarditis reported in the literature is quite rare; approximately $1 \%$ in accord with various studies. The view of the authors is that Group $G$ streptococcal endocarditis is often more aggressive, and is more frequently associated with embolic, neurologic and cardiac complications than endocarditis caused by viridans streptococci. We re- ported this case since Group G streptococci is unusual causative agent of infective endocarditis $(1,9,12,16,18)$.

\section{Case report}

A 29 year old female, was admitted to our hospital emergency department with the complaints of fever and loss of consiousness. It has been learned that she had had weakness, fatigue and diffuse myalgias for one week. On admission she was unconscious and was appeared malnourished. Her temparature was $38.5^{\circ} \mathrm{C}$, her pulse was 84 beats/min and her respiratory rate were 18 breaths/min, her blood pressure was $100 / 70 \mathrm{mmHg}$. Bilateral subconjunctival petechias were present. A grade 2/6 systolic murmur was audible at the left sternal border. There were left lower extremity paresis and right lower extremity plegia. The remainder of her physical examination yielded nothing remarkable. The patient had no significant medical history and in her family history her father had cardiac disturbances.

The laboratory investigation results were as follows: The leukocytes count was $14.7 \times 10^{9} / 1$; hematocrit level was $22.8 \%$, mean corpuscular volume was $84.3 \mathrm{fl}$, and platelet count was $96 \times 10^{9} / 1$. The peripheral blood smear showed $87 \%$ polymorphonuclear leukocytes, 7 \% lymphocytes, $6 \%$ monocytes. Erythrocyte sedimentation rate was $50 \mathrm{~mm} / \mathrm{h}$. Alanine transferase was $0.73 \mu \mathrm{kat} / 1$, aspartate amino- 
transferase was $0.78 \mu \mathrm{kat} / 1$, alkaline phosphatase was 1.83 $\mu \mathrm{kat} / 1, \gamma$ - glutamyl transpeptidase was $1.38 \mu \mathrm{kat} / 1$, lactate dehydrogenase was $5.02 \mu \mathrm{kat} / \mathrm{l}$, total bilirubin was 5.13 $\mu \mathrm{mol} / 1$, total protein was $51 \mathrm{~g} / \mathrm{l}$, albumin was $18 \mathrm{~g} / \mathrm{l}$, blood urea nitrogen was $3.99 \mathrm{mmol} / 1$, creatinine was $70.7 \mu \mathrm{mol} / 1$, sodium was $143 \mathrm{mmol} / 1$, potassium was $4.8 \mathrm{mmol} / 1$, chloride was $107 \mathrm{mmol} / 1$, protrombin time was 14.4 second. Urine analysis results were normal. Computed tomography of the brain (without contrast medium) was unremarkable. Chest radiography did not reveal any infiltrates.

A mildly turbid cerebrospinal fluid was yielded with lumbar puncture. When we analyzed it, glucose concentration was $3.55 \mathrm{mmol} / 1$, protein concentration was $290 \mathrm{mg} / \mathrm{l}$, and cell count was $57 \times 10^{6} / 1$ (90\% neutrophils, $10 \%$ lymphocytes). Gram stain and cultures of cerebrospinal fluid were negative.

The patient was admitted to infectious disease service with a presumptive diagnosis of central nervous system infection. After blood, urine, pharynx and cerebrospinal fluid specimens were obtained for culture, ceftriaxone $(2 \times 2 \mathrm{~g} /$ day, i.v.) was initiated empirically due to the leucocytosis and shift to the left.

In the cranial computerized tomography of the patient on the second day of hospitalization, only slight increase of contrast media uptake was observed in both parietal lobes, both frontal lobes and in the anterior areas of right occipital lobe.

Echocardiography had been done to the patient since the patient had cardiac murmur and signs of infection. On the echocardiography, there was a mass on the posterior surface of the mitral valve which was $2 \times 2.5 \mathrm{~cm}$ in length that might belong to a vegetation. According to the control echocardiographies, this mass was decreased in size.

Blood cultures became positive for group $G$ streptococcus and the organism was sensitive to penicillin, ceftriaxone, and vancomycin. In addition, the level of the complement 3 and 4 was normal. Thyroid function tests were normal. Brucella aglutination test was negative. VDRL test was negative. Tuberculin test was negative. ANA test was negative.

As a result, this case was accepted as infective endocarditis caused by group $\mathrm{G}$ streptococcus with multiple cerebral emboli. Gentamicin ( 2 x $80 \mathrm{mg} /$ day, i.v.) was added to the treatment at the eighth day of her hospitalization and Ceftriaxone was switched to $2 \times 1$ g/day,i.v. from $2 \times 2$ $\mathrm{g} /$ day,i.v. Ceftriaxone was given for 4 weeks and gentamicin was given for 2 weeks. The fever of the patient was begun to decline after the six days and progressive improvement of the patient's condition was seen. Cardiovascular surgeons did not think to operate the patient. The patient was followed by our clinic for 5 weeks and was sent to a rehabilitation center after antibiotic therapy.

\section{Discussion}

Group G streptococci may be found as a member of normal flora of the pharynx, intestine, vagina and skin. It may cause infections such as endocarditis, septic arthritis, pharyngitis, puerperal sepsis, peritonitis, pleuropulmonary infections, meningitis, cellulitis, otitis media and neonatal sepsis. The frequency of group $\mathrm{G}$ streptococcal endocarditis reported in the literature is quite low approximately $1 \%$ accordingly to various studies $(2,4,16)$. Blair et al. (3) reported that $\mathrm{G}$ group streptococci caused $8.4 \%$ of all beta hemolytic streptococcal endocarditis cases in accord to the published English language literature between year 1973 and 1987. Baddour et al also reported 3 cases as Group G streptococcal endocarditis (1).

The reported overall incidence of central nervous system complications of infective endocarditis varies greatly. In most series, the incidence of central nervous system involvement during the course of infective endocarditis is about $30 \%$ of all cases. The high rate of neurologic complications have been found in cases of infective endocarditis with left sided involvement. The two thirds of the case presenting with neurologic symptoms are due to major cerebral emboli and most commonly affect the middle cerebral artery and its branches. The remaining one third of cases the presenting signs and symptoms are seizures, meningismus, subarachnoid hemorrhage, intracerebral hemorrhage, subdural haemorrhage, personality change, visual disturbances, or weakness of the extremities. The patient who had neurological symptoms like purulent meningitis on admission had diagnosed as cerebral emboli. Group $G$ streptococcal endocarditis is often associated with embolic, neurologic and cardiac complications (8).

Cranial computed tomography or magnetic resonance imaging are the most useful diagnostic procedures in diagnosis of cerebral emboli. In the management of patients with infective endocarditis and cerebral emboli, optimal therapeutical strategy should be considered to prevent new emboli formation. The role of anticoagulant use is not clear. The role of cardiac surgery in the prevention of embolism in infective endocarditis is not widely accepted. The cardiac surgery is considered if vegatation is greater than one centimeter in diameter. In this case vegetatectomy or valve replacement may be tried (8).

In the treatment of infective endocarditis combination of cell wall active agents with an aminoglycoside is recommended at least during the first two weeks of therapy because non-group A $B$ hemolytic streptoccal strains may be less susceptible to penicillin than group $A$ hemolytic streptococcal strains. According to the data from a retrospective analysis of Group $G$ streptococcal endocarditis cases, combination therapy may diminish mortality rates. The optimal use of antibiotics and their effects remain controversial. There is little of informations in the literature about the in vitro antimicrobial bactericidal susceptibility patterns of Group $G$ streptococci, especially for nonpenicillin agents. The excellent bactericidal activity of penicillin $\mathrm{G}$ and 111.generation cephalosporins against Group $\mathrm{G}$ streptococci was shown in recent literature. Although in vitro antibiotic sensitivity against Group $G$ streptococci is 
excellent, the delayed clinical respons to antibiotic therapy is an interesting feature of group $\mathrm{G}$ streptococci $(1,7,12$, $15,16,17)$.

Ceftriaxone, a third generation cephalosporin, has an excellent in vitro activity against nonenterococcal streptococci. Ceftriaxone is both effective and safe for the treatment of streptococcal endocarditis due to the some studies performed with nonenterococcal streptococci. Ceftriaxone is also able to penetrate the cerebrospinal fluid that may be a better choice than the first generation cephalosporins as alternative regimens to penicillin when central nervous system complications were seen in infective endocarditis $(6,14)$.

Our patient was admitted to our service with a presumptive diagnosis of central nervous system infection. Ceftriaxone $(2 \times 2 \mathrm{~g} /$ day, i.v. $)$ was initiated empiricially due to the leucocytosis and shift to the left. In the cranial computerized tomography of our patient, minimaly contrast media uptake increased was observed in the bilateral parietal and frontal lobes and in the anterior areas of right occipital lobe. The blood cultures results were positive for group $\mathrm{G}$ streptococcus. In the echocardiography of the patient, there was a mass on the posterior surface of the mitral valve which was $2 \times 2.5 \mathrm{~cm}$ in length. It was thought that the echocardiographic appearence might belong to a vegetation. The patient was diagnosed as infective endocarditis according to the Duke criteria with the presence of two major and two minor criteria (including echocardiographic evidence, two persistently positive blood cultures yielding $\mathrm{G}$ Group beta hemolytic streptococcus, vascular phenomena, and fever $\left.>38{ }^{\circ} \mathrm{C}\right)$. Gentamicin $(2 \times 80 \mathrm{mg} /$ day, i.v. $)$ was added to the treatment at the eighth day of her hospitalization and Ceftriaxone was switched to $2 \times 1 \mathrm{~g} /$ day, i.v. from $2 \times 2 \mathrm{~g} /$ day, i.v. Ceftriaxone was given for 4 weeks and gentamicin was given for 2 weeks, and progressive improvement of our patient's condition was seen.

In summary, Group G streptococcal endocarditis is often more aggressive, and is more frequently associated with embolic, neurologic and cardiac complications. We reported this case because of its being an unusual form of infective endocarditis that was caused by Group G Streptococcus. We also want to emphasize that the patient who had neurological symptoms like meningitis had to be evaluated in details, in cerebral emboli cases, infective endocarditis should be encountered in differential diagnosis.

\section{References}

1. Baddour LM. Infective endocarditis caused by beta hemolytic streptococci. Infectious Diseases Society of America's Emerging Infection Network. Clin Infect Dis 1998;26(1):66-71.

2. Bayer AS, Scheld WM. Endocarditis and intravascular infections. In: Mandell GL, Bennett JE, Dolin R (eds) : Principles and practice of infectious diseases, 5th ed. Philadelphia: Churchill Livingstone Inc., 2000;857-903.

3. Blair DC, Martin DB. Beta hemolytic streptococcal endocarditis: predominance of nongroup A organisms. Am J Med Sci 1978;276:269-75.

4. Bucher A, Gaustad P. Septicemia and endocarditis caused by Group $\mathrm{G}$ Streptococci in Norwegian Hospital. Eur J Clin Microbiol Infect Dis 1990;4:251-56.

5. Durack DT, Lukes AS, Bright DK. Duke Endocarditis Service.New criteria for diagnosis of infective endocarditis: utilization of specific echocardiographic findings. Am J Med 1994;96:200-9.

6. Francioli PB. Ceftriaxone and outpatient treatment of infective endocarditis. Infect Dis Clin North Am 1993;17:97-115.

7. Francioli PB. Antibiotic treatment of streptococcal and enterococcal endocarditis: an overview. Euro Heart J 1995;16(Suppl. B):75-9.

8. Francioli PB. Complications of infective endocarditis. In: Scheld MW, Whitley RJ, Durack DT (eds): Infections of the central nervous system, 2nd ed. Philadelphia: Lippincott - Raven publishers, 1997:523-55.

9. Lam K, Bayer AS. Serious infections due to Group G Streptococci. Report of 15 cases with in vitro - in vivo correlations. Am J Med 1983;75:561-70.

10. Li JS, Sexton DJ, Mick N et al. Proposed modifications to the Duke Criteria for the diagnosis of infective endocarditis. Clin Infect Dis 2000;4:633-9.

11. Moreillon P. Endocarditis and endarteritis. In: Armstrong D, Cohen J (eds): Infectious Disease, 1st ed., Harcourt Publishers Ltd., 1999:2:50.1-10.

12. Rolston VIK. Group G streptococcal endocarditis and bacteremia. Am J Med 1987;82:187-8.

13. Sandre RM, Shafran SD. Infective endocarditis: Review of 135 cases over 9 years. Clin Infect Dis 1996;22:276-86.

14. Sexton DJ, Tenenbaum MJ, Wilson WR et al. Ceftriaxone once daily for four weeks compared with ceftriaxone plus gentamicin once daily for two weeks for treatment of endocarditis due to penicillin susceptible streptococci. Clin Infect Dis $1998 ; 27: 1470-4$

15. Shanson DC. New guidelines for the antibiotic treatment of streptococcal, enterococcal and staphylococcal endocarditis. J Antimicrob Chemothe 1998;42: 292-6.

16. Smyth EG, Pallett AP, Davidson RN. Group G streptococcal endocarditis: two case reports, a review of the literature and recommendations for treatment. J Infect 1988;16:169-76.

17. Traub WH, Leonhard B. Comparative susceptibility of clinical group A,B,C,F, and $\mathrm{G}$ beta-hemolytic streptococcal isolates to 24 antimicrobial drugs. Chemother 1997;43:10-20

18. Watsky KL, Kollisch N, Densen P. Group G Streptococcal Bacteremia. The clinical experience at Boston University Medical Center and a critical review of the literature. Arch Intern Med 1985;145:58-61.

Submitted January 2003.

Accepted May 2003.

Dr. Ilknur Erdem

Nuhkuyusu Cad.No: 153/4 Gencsuer Apt. 81150 Baglarbasi-Istanbul/ Türkey. e-mail: ilknurerdem@hotmail.com 